
acuñó. Contribuye así a construir la representación colonial de los indígenas como personas poco efectivas, lerdas, inferiores. Su perfil, que presentaré en este artículo, coincide bien con el de un agente colonizador.

PALABRAS CLAVE: Tráfico cultural, agente colonial, equivalencias interlingüísticas, sublación.

\title{
El difuso perfil de Juan de Betanzos como traductor de lenguas indígenas*
}

Lydia Fossa

Universidad Peruana de Ciencias y Tecnología (UPC), Lima, Perú.
From an indigenous perspective, Juan de Betanzos is not a trustworthy translator since his versions always served Spanish interests. He diminished words' contents and semantic coverage and, consequently, the culture that generated them. Thus, he contributed to the construction of a colonial representation of indigenous people as defective, inefficient, inferior. His profile, presented in this article, coincides with that of a colonial agent. KEYWORDS: Cultural trafficking, Colonial agent, interlinguistic equivalences, sublation. 
Los trabajos de Anthony Pym (2000) sobre la traducción y la interculturalidad me han recordado que una persona no sólo es lo que escribe, sino que deja pistas lingüísticas de su origen, su migración y su entorno en sus textos. Estas pistas pueden contribuir a aclarar las dudas que persisten acerca de Juan de Betanzos, autor de la Suma y narraçion (I55-1558), y aportar datos sobre sus experiencias lingüísticas. Sin duda, éstas influyeron en la calidad de sus traducciones.

La primera información léxica que ofrece este análisis es que el texto de Juan de Betanzos, escrito en castellano, está cargado de arabismos. Estos resultados fueron inesperados ya que tanto árabes como mozárabes y conversos tenían prohibido viajar a las Indias. Pero el léxico hispano-árabe de Betanzos, que verán más abajo, cuenta, hasta el momento, con 48 entradas identificadas en 304 páginas ( 153 folios). Esta terminología lo ubica en un área en la que árabes hispanizados y mozárabes, además de cripto-judíos y conversos ${ }^{\mathrm{I}}$ vivían en estrecho contacto con los españoles. Debo añadir que su léxico árabe es comparable al de Pedro de Cieza, escritor autodidacta contemporáneo suyo de origen converso, quien presenta 23 en 88 folios (205 páginas). Como contraparte, tenemos que escritores más ilustrados, que pasaron por la universidad, como Polo Ondegardo (jurista) y Pedro Sarmiento de Gamboa (cosmógrafo), presentan un tercio de los arabismos que apare-

* Este trabajo se basa en la ponencia del mismo nombre presentada en las VI Jornadas de estudio sobre pensamiento, cultura y sociedad colonial celebradas del $30 \mathrm{de}$ septiembre al 2 de octubre de 2004 en Ocucaje, Perú.

I «Los pobladores todos de las grandes ciudades cristianas, y los de las menos importantes, y los de los caseríos campestres, o sea, durante siglos, la inmensa mayoría de los habitantes de España y Portugal, quedaron hechos parte del mundo árabe. Todos ellos se arabizaron en la medida que quisieron...» (Alatorre, 1996: 77). cen en los dos primeros autores ${ }^{2}$. Es interesante notar que las etimologías de Corominas indican en varias ocurrencias que las palabras árabes que Betanzos utilizó pertenecen al registro vulgar. Covarrubias confirma este hecho en muchos de sus términos españoles. Esta información léxica, transferida a un paradigma social, colocaría a Betanzos, además, en un estrato social bajo, poco expuesto al mundo académico o letrado.

A pesar de la cantidad de términos árabes que ofrece, Betanzos no parece ser consciente de tener un léxico arabizado, ya que no lo indica a sus lectores. Esas palabras parecen haber sido completamente asimiladas, ya que el autor no las distingue de las derivadas del latín. Su grado de percepción lingüística podría haberse afinado durante su escolaridad formal en castellano o durante su entrenamiento como traductor, pero se sabe que su formación fue escasa y precaria. Pero, formalmente o no, aprendió a leer y escribir, como es el caso de Cieza. Este análisis indica también que llega a las Indias habiendo ya tenido una experiencia intercultural por su proximidad al mundo árabe, mozárabe o converso en la península ibérica, y de haber sido parte de una minoría social y lingüística.

Los antillanismos que presenta marcan también su pasado de migración transatlántica, con una posible estancia en esa zona. El número de entradas del kuná y taino quintuplica la terminología en uso común entre los españoles en los

2 He estudiado la Segunda parte de la Crónica del Perú de Pedro de Cieza, de familia de conversos y poca escolaridad. He encontrado II arabismos en las I 36 páginas de Notables daños de Polo Ondegardo, casi todos relacionados con la tributación y funcionarios de la administración pública, mostrando un uso tecnolectal de los arabismos que se insertan en el léxico de corte culto y educado que está, más bien, cargado de latinismos propios de su actividad en la jurisprudencia; Sarmiento de Gamboa en su Historia Indica, un texto cuidado y erudito, presenta 9 arabismos en I54 páginas. 
Arabismos en la Suma y Narración de Juan de Betanzos. Páginas 5 a 309

\begin{tabular}{|c|c|c|c|}
\hline & Páginas & Arabismos & Identificación lingüística \\
\hline I. & I03, I05, I8I, 304 & Acequia & $\begin{array}{l}\text { Corominas I983:24 } \\
\text { Covarrubias I995:I2 }\end{array}$ \\
\hline 2. & 268,275 & Adarga & $\begin{array}{l}\text { Corominas I983:27 } \\
\text { Covarrubias I995:I7 } \\
\end{array}$ \\
\hline 3. & $68,159,209$ & Adobados, adobar & $\begin{array}{l}\text { Corominas I983:28 } \\
\text { Covarrubias I995:19 } \\
\text { RAE I992:32 }\end{array}$ \\
\hline 4. & 76 & Adobes & $\begin{array}{l}\text { Corominas I987:28 } \\
\text { Covarrubias I995:19 } \\
\end{array}$ \\
\hline 5. & 32 & Adormido (mozárabe) & Alatorre I996:90 \\
\hline 6. & 84 & Ajuares & $\begin{array}{l}\text { Corominas I983:35 } \\
\text { Covarrubias I995:36 }\end{array}$ \\
\hline 7 & I57 & Albarradas [sic: albardas] & $\begin{array}{l}\text { Corominas I983:36 } \\
\text { Covarrubias I995:42 } \\
\end{array}$ \\
\hline 8. & $4 \mathrm{O}, \mathrm{I} 4 \mathrm{I}$ & Alborotado & $\begin{array}{l}\text { Corominas I983:37 Incierto } \\
\text { Covarrubias I995:45 }\end{array}$ \\
\hline 9. & 83 & alcuña $^{2}$ [sic: alcurnia $]$ & $\begin{array}{l}\text { Corominas I987:38 } \\
\text { Covarrubias I995:54 }\end{array}$ \\
\hline Io. & $\mathrm{I} 8, \mathrm{I} 4 \mathrm{I}$ & Alfiler & $\begin{array}{l}\text { Corominas I983:40 } \\
\text { Covarrubias I995:59 }\end{array}$ \\
\hline II. & 6I, 62, 244 & Algodon & $\begin{array}{l}\text { Corominas I987:4I } \\
\text { Covarrubias I995:6I }\end{array}$ \\
\hline I2. & I70 & Aljibes (variante vulgar) & $\begin{array}{l}\text { Corominas I983:42 } \\
\text { Covarrubias I995:65-66 }\end{array}$ \\
\hline I3. & 309 & Aljofar & $\begin{array}{l}\text { Corominas I983:42 } \\
\text { Covarrubias I995:66 }\end{array}$ \\
\hline I4. & 66 & Arrobas & $\begin{array}{l}\text { Corominas I983:64 } \\
\text { Covarrubias I995:I23 }\end{array}$ \\
\hline I5. & $\begin{array}{l}\text { I4, I7, 38, 57, 59, 60, 6I, 62, 72, } \\
75,77, \text { IOO, IIO, } 209\end{array}$ & Arroyo & $\begin{array}{l}\text { Corominas I983:64 Prerromano } \\
\text { Covarrubias I995:I24 }\end{array}$ \\
\hline I6. & $2 \mathrm{IO} 2 \mathrm{II}, 2 \mathrm{I} 3,227$ & $\begin{array}{l}\text { Atab [sic: atabal] (vulgar), } \\
\text { atabales }\end{array}$ & $\begin{array}{l}\text { Corominas I983:69 } \\
\text { Covarrubias I995:I33 hebreo }\end{array}$ \\
\hline I7. & $6 \mathrm{I}, \mathrm{I} 66,25 \mathrm{I}$ & Atambor(es) & $\begin{array}{l}\text { Corominas I983:555 } \\
\text { Covarrubias I995:134 }\end{array}$ \\
\hline I8. & $68, \mathrm{III}$ & Azotes, azotado & $\begin{array}{l}\text { Corominas I987:76 } \\
\text { Covarrubias I995:674 }\end{array}$ \\
\hline I9. & I99, 200 & Baldonando & $\begin{array}{l}\text { Corominas I983:8I } \\
\text { Covarrubias I995:I59 }\end{array}$ \\
\hline 20. & 60 & Barranca & Covarrubias I995:I68 \\
\hline $2 \mathrm{I}$. & $47,60,76,77$, I36, I49, 248 & Barro & $\begin{array}{l}\text { Corominas I983:87 Prerromano } \\
\text { Covarrubias I995:169 }\end{array}$ \\
\hline
\end{tabular}




\begin{tabular}{|c|c|c|c|c|}
\hline \multirow{23}{*}{54} & 22. & 77 & Bastardos & $\begin{array}{l}\text { Corominas I983:89 Incierto } \\
\text { Covarrubias I995:I7I }\end{array}$ \\
\hline & 23 & 304 & Borceguíes & $\begin{array}{l}\text { Corominas I983:102 Incierto } \\
\text { Covarrubias I995:199 }\end{array}$ \\
\hline & 24 & 264 & Calabazas & $\begin{array}{l}\text { Corominas I983:II7 Incierto } \\
\text { Covarrubias I995:23I }\end{array}$ \\
\hline & 25 & $\begin{array}{l}40,47,50,53,57,74,76, \\
\text { 103, I36, I70, I73 }\end{array}$ & Cantería, cantera & $\begin{array}{l}\text { Corominas I983:127 Incierto } \\
\text { Covarrubias 1995:257 }\end{array}$ \\
\hline & 26. & Io8 & Caracoles & $\begin{array}{l}\text { Corominas 1983:130 Incierto } \\
\text { Covarrubias I995:266 }\end{array}$ \\
\hline & 27. & 268 & Choza & $\begin{array}{l}\text { Corominas I983:198 Incierto } \\
\text { Covarrubias I995:392 }\end{array}$ \\
\hline & 28. & I4 & Destocado (toca) & $\begin{array}{l}\text { Corominas I983:570 } \\
\text { Covarrubias I995:923 }\end{array}$ \\
\hline & 29. & 68 & Enea [sic: anea] & Corominas I983:5I \\
\hline & 30. & 296 & Enfardelar (fardo) & $\begin{array}{l}\text { Corominas I983:268 posible árabe } \\
\text { Covarrubias I995:537 francés }\end{array}$ \\
\hline & 3I. & $270,27 \mathrm{I}$ & Escaramucearon & $\begin{array}{l}\text { Corominas 1983:242 Incierto } \\
\text { Covarrubias I995:490 }\end{array}$ \\
\hline & 32. & $\mathrm{I} 2, \mathrm{I} 3$ & Fulanos & $\begin{array}{l}\text { Corominas I983:284 } \\
\text { Covarrubias } 1995: 565\end{array}$ \\
\hline & 33. & 286 & Gaznates & $\begin{array}{l}\text { Corominas I983:295 mozárabe } \\
\text { Covarrubias I995:585 Incierto }\end{array}$ \\
\hline & 34 & IIO, 239, 296 & Hato, ato & $\begin{array}{l}\text { Corominas I983:315 } \\
\text { Covarrubias I995:623 Incierto }\end{array}$ \\
\hline & 35. & 86 & {$[\mathrm{H}]$ orones } & Corominas I983:325 mozárabe \\
\hline & 36. & I3, 89 & Izquierdo/a & $\begin{array}{l}\text { Corominas I983:340 vasco } \\
\text { Covarrubias I995:565 }\end{array}$ \\
\hline & 37 & $\mathrm{I} 42, \mathrm{I} 7 \mathrm{O}$ & Jarro/s & $\begin{array}{l}\text { Corominas I983:344 } \\
\text { Covarrubias I995:680 }\end{array}$ \\
\hline & 38. & 60 & Juncia & Corominas I987: 348 mozárabe \\
\hline & 39. & I8, IO2 & Losa, losas & $\begin{array}{l}\text { Corominas I983:366 Incierto } \\
\text { Covarrubias I995:72I }\end{array}$ \\
\hline & 40. & $89, \mathrm{II} 7, \mathrm{I} 70$ & Maroma & $\begin{array}{l}\text { Corominas } 1983: 383 \\
\text { Covarrubias } 1995: 738 \\
\end{array}$ \\
\hline & $4 \mathrm{I} \cdot$ & II4 & Mazorca & $\begin{array}{l}\text { Corominas I983:387 } \\
\text { Covarrubias I995:743 }\end{array}$ \\
\hline & 42. & I34 & Papagayo & Corominas I983:438 probable \\
\hline & 43 & 90 & Valde [en balde] & $\begin{array}{l}\text { Corominas I983:8I } \\
\text { Covarrubias I995:158-9 }\end{array}$ \\
\hline & 44 & $\begin{array}{l}39,46,68,79,95,96, \text { IIо, } \\
\text { II } 4 \text {, I16, I76, 210, 251, } 272\end{array}$ & Vasallaje, vasallo & $\begin{array}{l}\text { Corominas I983:598 celta } \\
\text { Covarrubias I995: } 95^{2-3}\end{array}$ \\
\hline
\end{tabular}




\begin{tabular}{|l|l|l|l|}
\hline 45. & $59,60,62,73$, I60 & Vera & $\begin{array}{l}\text { Corominas 1983:602 Incierto } \\
\text { Covarrubias 1995:958 }\end{array}$ \\
\hline 46. & 3 I, 38, 6I, II0, I84 & Zapatos (variante vulgar) & Corominas 1983:622 \\
\hline 47. & I64 & Zaque (variante vulgar) & $\begin{array}{l}\text { Corominas I983:622 } \\
\text { Covarrubias 1995:983 }\end{array}$ \\
\hline 48. & 77 & Zumo & $\begin{array}{l}\text { Corominas I983:626 } \\
\text { Covarrubias 1995:988 latín }\end{array}$ \\
\hline
\end{tabular}

Andes que no permanecieron allá3. Presenta dos nahuatlismos: galpón y petaca, que no aparecen en los textos de los demás autores estudiados. Cieza también ofrece dos nahuatlismos: mexicanos y tianguez, que no incluye Betanzos. Resulta interesante comentar que Cieza pasó aproximadamente trece años en regiones centroamericanas y en la costa de lo que hoy es Colombia, antes de su corta estancia en los Andes. Ondegardo no presenta ningún nahuatlismo y no permaneció en esas zonas.

Betanzos declara tener limitaciones lingüísticas cuando se lamenta ante el Virrey, a quien le dedica el texto, de tener que expresarse tal como lo hacían los nativos, y no en el castellano refinado en que le hubiera gustado hacerlo (1987: 7). Este podría ser un caso de exceso de humildad, pero también podría ser el reconocimiento de sus deficientes recursos lingüísticos. Muestro a continuación una lista de ejemplos seleccionados, sólo la letra «A», de barbarismos o vulgarismos:

Su escaso léxico dio pábulo a exhibir su léxico indígena andino de cerca de 500 términos, que hallarán completo en la internet: http://www. coh.arizona.edu/spanish/FossaLydia/Betanzos/

\footnotetext{
3 Polo Ondegardo, quien no se detuvo en América Central, sólo arroja los antillanismos ya asimilados al castellano y utilizados en toda la América española: aji, cacique, chicha y maiz. Véase el glosario bilingüe lenguas nativas-castellano de Polo Ondegardo extractado de su Notables daños de no guardar a los indios sus fueros en http:// www.coh.arizona.edu/spanish/FossaLydia/Ondegardo/ Ondegardo.html
}

betanzos.htm.

Betanzos era muy consciente de que los españoles no sólo necesitaban aprender las lenguas indígenas, sino también que debían saber cómo preguntar: «... como nuevos en el trato de los indios no sabrian inquirirlo y preguntarlo faltandoles la inteligencia de la lengua y los indios recelandose no osarian dar entera relacion...» (Betanzos, 1987: 9). Además, expresó la visión que tenía de su propio trabajo como traductor y de la calidad del mismo: «... el delicado y experimentado juicio de Vuestra Ilustrisima señoria requeria estilo gracioso y elocuencia suave lo cual yo para presente y servicio que yo a Vuestra Excelencia hiciese en mi falta y la historia de semejante materia no da lugar pues para ser verdadero y fiel traducidor tengo de guardar la manera y orden del hablar de estos naturales...» (Betanzos, 1987: 9). Se adhiere, así, a la perspectiva extranjerizante de la traducción que consiste en que la versión final le recuerde al lector que se trata de un texto que viene de otra lengua, de otra cultura. Betanzos reitera su posición extranjerizante: «... yo siendo mandado tengo de traducir como ello pasaba...» (Betanzos, I987: Io). Al anunciar que se ajustará a la traducción literal, repitiendo en una lengua romance, sintética, lo que se decía en una indígena, aglutinante, está renunciando a domesticar el texto (Schleiermacher, I992: 42; Venuti, I993: 210). Un texto «domesticado» es una versión que no tiene huellas de haber sido redactada en otra lengua ante- 


\begin{tabular}{|c|c|c|c|c|c|}
\hline \multirow{16}{*}{56} & & Página $^{3}$ & Antillanismo & Lengua & Identificación lingüística \\
\hline & I & 56 & aji & Taino de Santo Domingo & $\begin{array}{l}\text { Corominas I983:34 Alvar } \\
\text { I970:45-46 }\end{array}$ \\
\hline & 2 & 368 & bejucos & Taino & Corominas I983:9I \\
\hline & 3 & $6 \mathrm{I}$ & cabuya & Taino de Santo Domingo & Corominas I983:II4 \\
\hline & 4 & $6 \mathrm{I}$ & caciques & Taino de Santo Domingo & $\begin{array}{l}\text { Corominas I983:II4 } \\
\text { Alvar } 1970: 55-56\end{array}$ \\
\hline & 5 & 6I & chicha & $\begin{array}{l}\text { Parece ser voz de indios } \\
\text { cunás, Panamá }\end{array}$ & Corominas 1983:194 \\
\hline & 6 & 192 & Galpon & Azteca [nahuatl] & Corominas I983: 289 \\
\hline & 7 & $2 \mathrm{OI}$ & gaobo [caoba] & Taino de Santo Domingo & Corominas I983:I28 \\
\hline & 8 & 250 & hamacas & Taino de Santo Domingo & $\begin{array}{l}\text { Corominas I983:314 } \\
\text { Alvar } 1970: 67-68\end{array}$ \\
\hline & 9 & 63 & Maiz & Taino de Haití & $\begin{array}{l}\text { Corominas I983:374 } \\
\text { Alvar I970:76-77 }\end{array}$ \\
\hline & IO & $\mathrm{I} 34$ & micos & $\begin{array}{l}\text { Prob. del caribe de Tierra } \\
\text { Firme }\end{array}$ & Corominas I983: 395 \\
\hline & II & 264 & petacas & Azteca [nahuatl] & $\begin{array}{l}\text { Corominas } 1983: 455 \\
\text { Alvar } 1970: 87\end{array}$ \\
\hline & $\mathrm{I} 2$ & I & Peru & $\begin{array}{l}\text { Incierta (sur de Panamá, } \\
\text { norte de Ecuador) }\end{array}$ & $\begin{array}{l}\text { Covarrubias I995:8I8 } \\
\text { Fossa } 2003\end{array}$ \\
\hline & I3 & 69 & tunas & Taino de Haití & $\begin{array}{l}\text { Corominas I983:589 } \\
\text { Alvar I970:IOI-IO2 }\end{array}$ \\
\hline & I4 & I63 & xaqueyes [xaguey] & Taino de Santo Domingo & Corominas $1983: 342$ \\
\hline & I5 & $\mathrm{I} 34$ & yuca & Taino de Santo Domingo & $\begin{array}{l}\text { Corominas I983:6r6 } \\
\text { Alvar Io70:I04 }\end{array}$ \\
\hline
\end{tabular}

\begin{tabular}{|c|c|c|c|c|}
\hline & Página & Castellano & Registro & Fuente \\
\hline I. & 275 & aforia & & No la registran los diccionarios consultados. \\
\hline 2. & 37 & agora & Hoy vulgar & $\begin{array}{l}\text { DRAE } 42 \\
\text { Covarrubias } 26\end{array}$ \\
\hline 3. & I76 & aliende & Por allende & DRAE $_{72}$ \\
\hline 4. & I76, I97 & ampliedad & & No la registran los diccionarios consultados. \\
\hline 5 . & 37 & aquestos & Hoy sólo en poesía & $\begin{array}{l}\text { DRAE I24 } \\
\text { Covarrubias Io9 }\end{array}$ \\
\hline 6. & 233 & aturado & Usase en Salamanca. & DRAE I6I \\
\hline $7 \cdot$ & 287 & ayna & Bárbara & $\begin{array}{l}\text { DRAE }_{5 \mathrm{I}} \\
\text { Covarrubias } 34\end{array}$ \\
\hline
\end{tabular}


riormente. Pero tengo dudas de si al decir que su intención era «...guardar la manera y orden del hablar de estos naturales...» (Betanzos, 1987: 9) sólo está justificando las limitaciones de su castellano, puesto que una traducción literal entre esos dos tipos de lenguas, sintéticas y aglutinantes, es imposible. Y, por si acaso alguien lo acusara de malinterpretar a sus informantes o de dar descripciones falsas, desestimó esas inminentes acusaciones como «antojos o sueños» (Betanzos, 1987: 9). En su defensa, indicó que sabía la lengua indígena mejor que los primeros «lenguas», estableciendo con su afirmación su participación en la segunda generación de intérpretes en los Andes, la de la década del I540, aquellos de origen peninsular que habían reemplazado a los indígenas, tal como lo reportara al Rey, desde Lima, el Licenciado Martel de Santoyo en 1542 (Lisson-Chaves, I943: II2)4.

Así, el texto que dijo haber traducido literalmente está cuajado de palabras y frases que mantuvo en la lengua fuente:

\begin{tabular}{|l|c|}
\hline Palabras y frases indígenas seleccionadas & Págs \\
\hline - Acocapa Ynga aucay quita atixu & \\
- llacxaimoctiangui cuna punchaupi & 32 \\
- Aguacolla quisca & 77 \\
- Allapo coiquis & $7 \mathrm{I}$ \\
- Apo Ynga Randirimaric & III \\
- Apoyupangue Uxuta Urcoguaranga & 25 \\
- Caiñoc aprandicanga caiño & \\
caprandicachun & I3I \\
\hline
\end{tabular}

Acompañó las frases y palabras indígenas con diversos tipos de equivalencias 5 bilingües, de

\footnotetext{
4 «Las lenguas o ynterpretes que ay en esta tierra son yndios naturales della que saben algo de la lengua española e asta oy no a avido español ninguno que sea ynterprete, ni esto se ha procurado de que ay ynconveniente... Vuestra magestad mande que de aquí adelante los ynterpretes sean españoles...» (Lisson-Chaves, I943: II2).

5 Aquí sigo la clasificación que propone Enguita Utrilla en su artículo de 1979.
}

manera que es evidente que quería que el texto fuera claro y comprensible para sus lectores. ¿Por qué entonces conservó palabras en la lengua original? ¿Exotismo? Betanzos no se adhirió a ninguno de los anuncios que hizo en la Suma y narración en cuanto a la traducción y la codificación.

Desde I970, José M. Enguita Utrilla ha estudiado la presencia de lo que llama «indoamericanismos léxicos» en documentos y crónicas españoles, estableciendo claramente su perspectiva hispánica sobre el tema. Hace contribuciones verdaderamente interesantes al estudio y el análisis de la traducción del siglo xvi, tal como la realizaban individuos semicultos españoles que se lanzaron a escribir historias, crónicas e informaciones. Enguita propone varios procedimientos a través de los cuales los españoles descifraban la semántica contenida en «vocablos bárbaros» (Enguita, 1979: 288):

\begin{tabular}{|l|}
\hline José M. Enguita Utrilla (1979: 288) \\
\hline Procedimientos \\
Descripciones \\
- Definiciones \\
- Explicaciones \\
- Coordinaciones interlingüísticas \\
- disyuntivas \\
- copulativas \\
- Traducciones \\
- Caracterización indirecta \\
\hline
\end{tabular}

Estos procedimientos se utilizaban para acercar los textos que describían el mundo indígena a los lectores españoles, europeos. La traducción que primó en este tipo de textos «laicos» fue de las lenguas indígenas al castellano. Pero, en las principales traducciones de textos doctrinarios del castellano o del latín a las lenguas indígenas, se pueden identificar otras estrategias, además de los procedimientos que menciona 
Enguita y que han sido estudiados por César Itier. Son indicativos de una aproximación más compleja tanto al original como al texto traducido. Itier no las llama «procedimientos» sino «manipulaciones» ${ }^{6}$ porque las transformaciones impuestas a las lenguas indígenas las forzó a decir lo que sus hablantes nunca habían siquiera pensado, exigiéndole tanto a la semántica como a la morfología expresar conceptos europeos (1995: 321-322).

César Itier (1993, 1995)

\section{Manipulaciones}

- Préstamos (renuncia a traducir)

- «Traducción»

- Equivalencias

- Sintagmáticas

- Contextuales

- Perífrasis

- Neologismos

- Composición

- Derivación

- Sustituciones

- Significantes

- Significados

Los efectos de estas manipulaciones no sólo cambiaron la lengua, sino que crearon giros, combinaciones y contextualizaciones que eran incomprensibles para los hablantes nativos7.

Nicanor Domínguez (1994) y yo (en prensa) creemos que Betanzos se inició como traductor en el seno de la Orden Dominica en los Andes. A los lexicógrafos de la Orden de Predicadores

6 «... la tradición oral y la lengua quechuas fueron objeto, por parte de la Iglesia peruana colonial, de una minuciosa e inteligente empresa de manipulación» (Itier, I993: 172).

7 Véase Rafael (1993) para el caso de las Filipinas.

les preocupaba que el quechua y otras lenguas indígenas fueran incapaces de expresar las sutiles, sofisticadas, ideas españolas sobre el catolicismo. Algo muy diferente ocurría con las lenguas hebrea, árabe, latina y romance que se bastaban para describir religiones muy similares, que venían del tronco común de la judía. Las lenguas indígenas fueron consideradas limitadas puesto que no tenían el vocabulario apropiado para lograr una expresión inteligible del catolicismo ortodoxo. Por ello, se asumía que siempre sería necesario, y hasta provechoso, manipularlas.

En Narrativas problemáticas: Los Inkas bajo la pluma española (Fossa 2006) he estudiado el grado de conocimientos que tenía Betanzos del quechua, basándome en su uso de los posesivos. Llegué a la conclusión de que éste no había sido ni tan avanzado ni tan sistemático como se había afirmado. Mi estudio se basó en varios ejemplos similares a este:

\begin{tabular}{|l|l|l|}
\hline Página & Folio & Cita \\
\hline 220 & Io6r & $\begin{array}{l}\text { Ynga Guauquin que dice } \\
\text { el hermano del Ynga }\end{array}$ \\
\hline
\end{tabular}

Utilizaré el mismo ejemplo para iniciar el análisis del trabajo de traducción de Juan de Betanzos. De acuerdo a Enguita, el procedimiento de traducción utilizado por Betanzos sería el de la Definición, «fácil y exacta percepción del concepto correspondiente a las voces que las originan. En su construcción destaca la brevedad» (I979: 288). A primera vista, eso es lo que parece estar sucediendo en este ejemplo. Comenzaré con los aspectos gramaticales de la frase en quechua. Uno de los posesivos, cuando se le requiere, lleva dos marcas sufijales, una en el posesor, _ $p, y$ otra en lo poseído,_n.En el presente ejemplo, lo poseído lleva la marca sufijal_n, no así el posesor, que carece de marca. La forma correcta sería: Yngap Guauquin. Esta omisión se debe probable- 
mente a la influencia de la fonética castellana, en la que es inusual que una palabra finalice en una oclusiva labial. También hay que tener en cuenta el sustrato morfosintáctico del castellano, que no exige una indicación gráfica en el objeto poseído. La marca es léxica: la palabra «del» es suficiente para indicar pertenencia; y sintáctica: el objeto la antecede y el sujeto la sigue.

Observando ahora su aspecto semántico, el significado quechua de guauque /wawqi/, no es simplemente «hermano» ${ }^{8}$. También quiere decir pariente y aliado, de acuerdo con Domingo de Santo Tomás (1951: 289)9. Para el lexicógrafo quechua Anónimo, esta palabra tiene aún más significados y más complejos, además de los de vínculos sanguíneos o amicales: "Huauque dezían a la estatua o ydolo particular que tenia cada nación.» (I95I: 50). En aimara, Ludovico Bertonio ofrece: «Huauque, kochomasi: amigo» (I879: 154). Así es que en la frase del ejemplo: «Ynga Guauquin que dice el hermano del Ynga» guauque se referiría a un ser humano vivo, o a la representación de uno mismo $^{\mathrm{IO}}$ o de toda una nación a través de un obje-

8 En castellano, los significados de «hermano» están limitados al campo del parentesco (legítimo o ilegítimo), y al de las órdenes religiosas (el último como una extensión del primero). Así aparece en el Tesoro de I6II de Covarrubias (629), y en la versión del siglo xxi del Diccionario de la lengua española (I992: 774).

9 "Guauquine, hermano generalmente. Guauquine, primo hermano o pariente cercano. Guauquine ayllo, cercano en sangre. Guauquinchacuni, gui, hacerse hermanos o amigos. Guauquinacoc, aliados en amistad assi» (Santo Tomás, I95I: 289).

Io La cita completa dice así: «...teniendo esta nueva y se viese señor mando luego hacer un bulto de sus mismas uñas y cabellos el cual imitaba a su persona y mando que se llamase este bulto Ynga Guauquin que dice el hermano del Ynga y este bulto ansi hecho mando que fuese puesto en unas andas y mando a un criado suyo que se decia Chima que dando a este bulto que le sirviese y que tuviese cargo de guardarle y mirarle y dando a este bulto otros muchos mozos y servicio mando que luego fuese...llevado en sus andas por la posta a do sus capitanes estaban Chalcuchima y Quizquiz para que las provincias y gentes que sujetasen diesen obediencia a aquel bulto en lugar de su persona to (piedra o bulto). Pero, al tener en cuenta todas las definiciones, se observa que guauque tenía un significado que estaba más cerca a alter ego que a «hermano» de sangre o de orden.

E1 rico campo semántico cubierto en el quechua por la palabra guauque queda reducido al del castellano, restringido a humanos, a individuos. Las dos palabras, guauque y «hermano» comparten algunos rasgos de significado; el problema es que una vez que el término ha sido traducido al castellano no se ha dado una explicación que indique la reducción semántica del quechua, ni se le ha añadido una paráfrasis que la compense. Guauque se admite, para los hablantes de castellano, como un perfecto sinónimo de "hermano», perdiendo así tanto rasgos inherentes como aferentes en el proceso de traducción al castellano. En general, los items del léxico indígena, $y$, en particular, las palabras en quechua, pierden una parte importante de su contenido semántico además de casi todo su contenido simbólico en el proceso de traducción de Betanzos. Utilizando la terminología de Itier, lo que ha tenido lugar en este caso es una sustitución de significante con eliminación de parte del significado.

Pym nos proporciona un modelo de intersección (2000: 6) que muy bien podría ilustrar esta situación. En mi ejemplo, el contenido de «hermano» está considerado dentro del de «guauque». Todo el ámbito de significado de "guauque» que excede el de «hermano» no entra en la «equivalencia» guauque-hermano; es decir, la paridad o equivalencia con el término extranjero se basa solamente en los rasgos de la palabra castellana que intersectan, omitiéndose los rasgos semánticos indígenas que exceden la intersección.

y ansi fue... llevado y dado a los capitanes los cuales... hicieronles... muy grandes sacrificios y ansi servian y respetaban a este bulto como si fuera alli en persona el mesmo Atagualpa...» (Betanzos, 1987: 220). 
Al dibujar las formas intersectantes de Pym para ilustrar el resultado de esta equivalencia, la que representa a guauque resulta ser significativamente mayor que la que representa la palabra «hermano». Vemos en el gráfico que guauque cubre y excede ampliamente el campo semántico de «hermano».

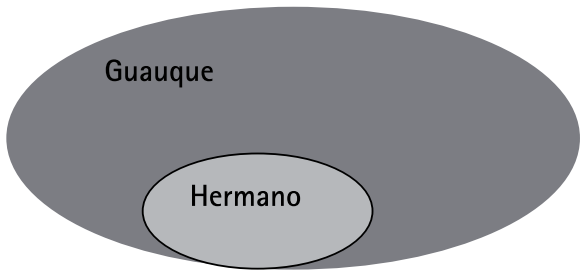

Todo ese «exceso» de significado, excesivo desde la perspectiva española, se pierde en la versión de Betanzos. Para Itier, éste sería un caso en que «las realidades concebidas por los andinos antiguos se encuentran sustituídas, a través del lenguaje, por realidades europeas» (Itier, I995: 325, mi traducción).

Otros autores, desde una perspectiva postcolonial, han elaborado teorías sobre hechos de traducción similares. Mary Louise Pratt describe hechos interlingüísticos semejantes como «tráfico» y no como traducción $(2002)^{\mathrm{II}}$. Para mí ha tenido lugar una «reducción», una banalización ${ }^{12}$ y simplificación de los términos

II «La traducción es una metáfora profunda pero incompleta del tráfico de significados. Probablemente no sea, a largo plazo, una base adecuada sobre la cual asentar una teoría de la elaboración de significados interculturales, y ciertamente no es un sustituto de tal teoría. Pero, explorar esa metáfora puede ser una manera productiva de clarificar lo que tal teoría podría ser. La traducción puede recordarnos constantemente que el estudio de la mediación cultural será tanto una ciencia como una poética» (Pratt, 2002: 35, mi traducción).

${ }^{\text {I2 }}$ «En este contexto, la traducción tiene una definición especial, tal como lo explica Tejaswini Niranjana, que refleja la lucha por el poder en la que tiene lugar. Así, sería más apropiado referirse a la traducción como 'sub- y conceptos en quechua, creando la situación para la sublación Niranjana 1992) ${ }^{\mathrm{I3}}$, y no para la traducción, con la consiguiente subalternización de la cultura. Venuti también ha estudiado la problemática de la traducción en contextos coloniales y enfatiza lo que es «el poder de la traducción para reconfigurar y rebajar los textos extranjeros, para trivializarlos y excluir las culturas foráneas...» (1993: 208, mi traducción). Según mi definición de trabajo, la traducción colonial es una traducción de lenguas de valor y prestigio desiguales, en la que la lengua colonizada queda explicada y asimilada a la lengua colonizadora.

En la cita siguiente, quiero resaltar las características de la traducción cultural colonial, sin dejar de subrayar los esfuerzos del autor por transmitir el contenido y el contexto de una realidad desconocida.

\begin{tabular}{|c|c|l|}
\hline Página & Folio & $\begin{array}{l}\text { Cita } \\
\text { «... y las mujeres salieron ansi } \\
\text { mismo vestidas muy ricamente } \\
\text { con unas mantas y fajas que ellos } \\
\text { llaman chumbis muy labradas de } \\
\text { oro y con los prendedores de oro } \\
\text { muy fino los cuales son los unos } \\
\text { alfileres largos de dos palmos que } \\
\text { ellos llaman topos...» }\end{array}$ \\
\hline
\end{tabular}

ducción' (sublation en inglés). Por sub-ducción me refiero al proceso de simplificación, reducción y banalización de los significados y de los contenidos semánticos que tiene lugar cuando se traducen lenguas colonizadas a la lengua del colonizador. Así, la sub-ducción también confirma el menor prestigio que el colonizador le atribuye a las lenguas nativas ya que éstas no pueden expresar sus ideas 'exactamente'» (Fossa, 1998: 163).

r3 Niranjana define la traducción en un contexto colonial como si ésta fuera un proceso de 'sublation', esto es, literalmente, como una sub-traducción. Con ese término ella identifica el proceso de simplificación, reducción de contenidos semánticos, así como la banalización de los significados que tiene lugar al traducir lenguas colonizadas a la lengua del colonizador. 
En este pasaje, Betanzos se refiere a prendas femeninas. Utiliza dos palabras en quechua: chumbis /chumpi/, y topos/tupu/. El primer término es algo ambiguo ya que al decir «con unas mantas y fajas que ellos llaman chumbis», no queda claro si chumbis se refiere a ambas, «mantas y fajas», o solamente a la última palabra. De acuerdo a Santo Tomás, chumbi es «ceñidero»; y chumbi o guachuco es «faxa para ceñirse» (1951: 273). El Anónimo tiene primero chumpit como «color castaño» (I95I: 29), y en una segunda entrada para chumpi, consigna el significado de «faxa de Indias, o cingulo». «Faxa», «ceñidero», y «cingulo» significan básicamente lo mismo: cinturón. El Anónimo confirma el uso de chumpi solamente para mujeres, quienes utilizaban estas bandas alrededor de sus cinturas para mantener sus otras prendas en su sitio. Betanzos dice que estos chumpi eran especiales, ya que estaban trabajados en hilo de oro. Los describe detalladamente para mostrar que el oro estaba presente en todas partes, incluso en la ropa femenina. Ve otros objetos de oro: los topos o /tupu/. Además de indicar que estaban hechos de oro muy fino, le añade medidas a la descripción («dos palmos») para destacar la magnitud y el rango de la utilización de los metales preciosos.

$\mathrm{El}$ resaltar el oro presente en las prendas nos distrae, y también al autor, del uso ceremonial de estos objetos, e incluso de la ceremonia misma que contextualiza el uso de esas prendas. Las festividades se relacionan con los inicios de la cultura Inka, reinstaurando la emergencia de las parejas inaugurales de la cueva de Pacaritampu. Betanzos sólo nos está dando las características particulares de los objetos que le llamaron la atención, desde una perspectiva europea, y no el aspecto de más importancia general tal como se percibía desde

I4 La sonorización /b/ de la oclusiva sorda / $/ \mathrm{p}$ es característica del dialecto chinchay del quechua (costa y sierra centrales). La oclusiva sorda se utiliza en dialectos sureños. una perspectiva indígena. Lo que estamos viendo acá no es sólo una «reducción» de los significados quechua (Fossa 1998) ${ }^{15}$, sino también un cambio de enfoque de las vestiduras especiales para una cere61 monia particular a la presencia de oro en esas vestiduras. Este último procedimiento de traducción es tan importante como el primero; el primero se refiere al contenido semántico, y el segundo al punto de vista que, en este caso, pone de relieve un aspecto importante para la lengua meta que no se destaca en la lengua fuente. El efecto de un cambio de enfoque «subrepticio» en una traducción tiene un impacto determinante sobre la idea que se hace el lector del valor cultural de la sociedad ${ }^{\mathrm{r} 6}$. Se ha dado lo que Venuti llama «transgresión de los valores discursivos» (I993: 209 $)^{\text {r7 }}$. Quiero subrayar que la perspectiva europea del autor es la que prevalece, en detrimento de la perspectiva indígena, que queda relegada sin mayor explicación. Además, refleja el escaso prestigio que tienen la lengua y la cultura indígenas para el colonizador/traductor.

En el siguiente ejemplo:

\begin{tabular}{|c|c|l|}
\hline Página & Folio & Cita \\
\hline Io9 & $54 \mathrm{~V}$ & $\begin{array}{l}\text { «... que fuesen señaladas ciertas } \\
\text { Cozcoynacacuna que dice como } \\
\text { decimos ciertas matronas roma- } \\
\text { nas...» }\end{array}$ \\
\hline
\end{tabular}

I5 «Mi opinión es que la Segunda Crónica de Cieza contiene información semántica perteneciente a los términos quechua y que debe tomarse en consideración para dar cuenta, por lo menos en parte, de la «reducción» de que han sido objeto, al incorporársele como lo «nuevo» a la logósfera europea. También podemos observar allí la transferencia de rasgos sémicos del castellano al quechua, rasgos que no le eran propios a los hablantes de quechua en contextos americanos») (Fossa, I998: 7 en mi ms).

I6 «... la traducción ejerce un poder enorme en la construcción de las identidades nacionales para las culturas extranjeras y por lo tanto puede tener un papel importante en los conflictos raciales y étnicos y en las confrontaciones geopolíticas» (Venuti, I993: 209, mi traducción).

I7 Mi traducción de: «transgression of discoursive values» (Venuti, 1993: 209). 
tenemos que esta palabra, Cozcoynacacuna, es en realidad, dos: Cozco e yñacacuna y significa «las yñacacuna del Cuzco». Se podría pensar que yñaca es quechua ya que está asociada al Cuzco, pero ni Domingo de Santo Tomás ni el Anónimo incluyen esa palabra en sus Vocabularios. Bertonio lo hace, así que podemos pensar que yñaca es aimara. Dice: «Iñaca, vel Palla; Muger que viene de casta noble de los Ingas» (I879: I75). Bertonio siente la necesidad de incluir el equivalente interlinguístico, palla (quechua), sugiriendo que yñaca sería puquina. El Anónimo ofrece el mismo significado para Palla en quechua: "India noble, señora de linage de Ingas» (1951: 67) que Bertonio le da a «yñaca». El sufijo_cuna es un pluralizador en quechua. De manera que lo que tenemos acá es una palabra aimara o puquina tratada por Betanzos como si fuera una palabra quechua. En este sentido, tenemos una asimilación del aimara o del puquina al quechua, de lo que no nos informa el autor.

Además, tenemos lo que yo llamo una «saturación» de significados. Muchos aspectos de la vida Inka han sido comparados a los de los romanos, como sus caminos y sus edificios, su conjunto de deidades y sus rituales. Esas similaridades, reales o imaginadas, los llevó a describir el Tawantinsuyu como un «imperio» y a las «señoras de linage de Inkas» como «matronas romanas». Esta equivalencia excede el campo de la demografía e invade el de lo político.

No es este un error de percepción; es una estrategia de transmisión de lo raro o desconocido por asociación con lo conocido o lo familiar. Pero, no permite el establecimiento de relaciones de identidad con los contenidos semánticos quechua: constituye una distorsión que acerca el contenido a constructos con los cuales coinciden sólo en parte (Fossa, I998: Io). Pero Betanzos pensó que sus lectores enten-

derían la función que cumplían estas mujeres en el estado Inka, tal como él lo hizo, a través de ese filtro conceptual. El efecto final de esta estrategia es vaciar el significado de yñaca tal como se le entiende en la cultura que lo generó y lo utilizó, y rellenarlo con rasgos semánticos y culturales foráneos. Esta intersección particular se observa en el siguiente gráfico, en el que se puede apreciar que el término indígena comparte gran parte de su contenido con el término español, pero tiene rasgos especificos que se ignoran al momento de la explicación. Asimismo, los rasgos semánticos de «matronas romanas» exceden largamente el ámbito significativo de yñacacuna:

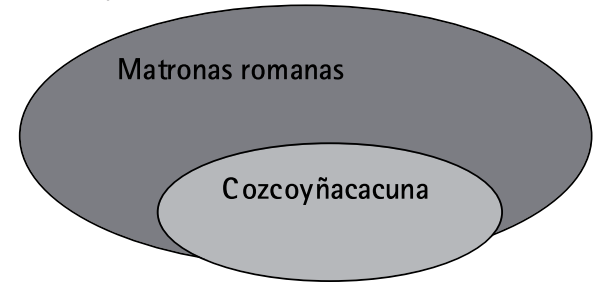

Esto resulta en una disminución del contenido semántico intrínseco, propio, y una superabundancia de significado añadido, extrínse$\mathrm{co}^{\mathrm{I} 8}$. Para Enguita, esto sería una explicación, y para Itier, una perífrasis con transformación de sintagma por semejanza en los contenidos interlingüísticos.

\section{CONCLUSIONES}

Es difícil afirmar, dada la escasa información disponible, el origen étnico de Betanzos. Pero la

I8 «De esta manera, no sólo se han perdido los significados y los rasgos del quechua (así como de otras lenguas vernáculas), sino que a los lexemas nativos se les ha atribuido rasgos semánticos que no son propios de las condiciones de comunicación y de prágmaticas andinas» (Fossa, 1998: Io). 
evidencia lingüística de su castellano apunta a una base pluriétnica, pluricultural, que vincula aspectos árabes, mozárabes y conversos con un castellano con registros vulgares. Estos factores léxicos lo sindican como miembro de un grupo social y racial mixto, y a un estatus social bajo. Al castellano de Betanzos también le falta lustre y amplitud a su vocabulario, confirmando su registro como el producto de una escasa escolaridad formal. Estas limitaciones conciernen a la expresión escrita y, por inducción, a la hablada, ya que exhibe una literariedad muy marcada por lo oral ${ }^{\mathrm{I} 9}$.

Los antecedentes pluriculturales peninsulares de Juan de Betanzos se ven enriquecidos con el vocabulario indígena americano del área caribeña (nahuatl, taino y kuna) y de los Andes (quechua, aimara y puquina). El autor no es consciente de las diferencias entre lenguas caribeñas y andinas ${ }^{2 \circ}$, y dentro de esta última categoría, no es capaz de discernir entre lo que él llama quechua y el aimara, y aún el puquina, tal como lo muestran sus textos. Con la evidencia hallada hasta ahora sólo se puede confirmar su poca destreza en la lengua quechua. A esto se le añade el contenido de la misiva del Licenciado Martel de Santoyo que indica claramente que tanto el clero como los legos (Betanzos entre ellos) hicieron muy pocos esfuerzos por aprender lenguas nativas antes de 1542 (LissonChaves, I943: I00) ${ }^{21}$.

I9 «El discurso oralizante se organiza alrededor de un registro minucioso de las diferencias, por momentos desordenado o confuso, por momentos distorsionado, según la cualidad de la imaginación o la memoria de los informantes/autores andinos y según la experiencia que haya alcanzado el traductor/autor europeo o nativo. Uno de los problemas que plantea la asociación de la oralidad con la escritura es el de la naturaleza de la interacción y de los elementos que marcan las diferencias entre dichas prácticas» (Jákfalvi-Neira, I993: 259).

20 Betanzos nunca señala la procedencia de la palabra indígena que utiliza.

${ }_{21}$ «... hasta oy en ningun pueblo desta tierra se sabe
La habilidad de Betanzos como traductor cultural y linguístico es más difícil de evaluar cuando se compara sus propósitos declarados con sus resultados. Utiliza una amplia gama de estrategias para traducir las formas lingüísticas, más variadas y más complejas que las que se encuentran en la lista proporcionada por Enguita; más cerca de lo concebido por Itier. Pero Betanzos no sigue consistentemente ninguna estrategia de traducción, yendo de una aproximación extranjerizante declarada a plasmar una versión domesticada, europeizada, de la vida Inka.

Sucede que Betanzos es incapaz de cumplir con el rol de traductor cultural debido a su ignorancia o falta de interés en las culturas y lenguas indígenas. Cuando la ignorancia está en juego, se explican los casos de subtraducción o sublación. Si predomina la falta de interés, los casos de sobre-traducción pasan desapercibidos por el traductor que se preocupa más por facilitarle la comprensión al lector que por transmitir las características de la otra cultura. Las instancias en las que tiene lugar un cambio de enfoque caen también en la categoría de las traducciones opacas ya que el énfasis en el significado cambia, asumiéndose el de la cultura del traductor sin alertar al lector. Betanzos no da cuenta de la suma y resta del contenido semántico que sufren las lenguas nativas al ser traducidas al castellano, pero esto sucede constantemente: algunas palabras quedan sin parte de su contenido semántico mientras que otras quedan vaciadas

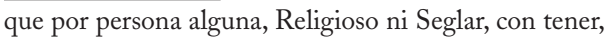
como todos tienen, yndios, asy conventos de frayles como prelado y algunos clerigos, se aya entendido en la dicha conversion ni doctrina ni dadose a saber vocablos de la lengua natural para ello ni desto a avido mas memoria que de una cosa digna de abominacion, aunque vocablos para pedirles oro e negociar en los casos que arriba digo no ay poca destreza» (Lisson-Chaves, I943: I00). 
de él y repletas de significados de la lengua meta.

Al realizar traducciones culturales, Betanzos 64 «estafa» a las lenguas indígenas constantemente ya que no se inmuta al dar equivalencias interlingüísticas como si hubiera una perfecta sinonimia entre los términos escogidos de ambas lenguas como idénticos. El aspecto más importante de la traducción cultural, la permanencia del simbolismo expresado con los semas aferentes, está ausente. La contextualización puede contribuir a mantenerla, así como los elementos explicatorios que provienen de un exhaustivo conocimiento de la cultura que produce los rasgos cargados de simbolismo de ciertas expresiones. Pero Betanzos no se detiene a proporcionar el ambiente cultural que enmarca y valida las expresiones andinas.

En general, sus traducciones son más indicadoras de un tráfico cultural que de otra cosa: Betanzos percibió los aspectos culturales nativos que le podrían servir en sus afanes pecuniarios, de manera que centró su atención, más que en la cultura misma, en los asuntos que lo beneficiarían más. Betanzos disminuye el contenido y el rango de palabras y frases y de la cultura que las acuñó, contribuyendo a construir la representación colonizada de los pueblos indígenas como defectivos, bastos, inferiores. La representación de este «traductor» ciertamente coincide con la descripción de un agente de colonización, ya que sus versiones siempre están al servicio de los intereses españoles.

Juan de Betanzos pertenece a la segunda generación de intérpretes y traductores formada por españoles que sabían algo del quechua general y por mestizos, que eran parcialmente bilingües. Betanzos llegó a ser, en su madurez, intérprete oficial de la Audiencia de Lima. Aún así, la descripción que Garcilaso hace de su habilidad como traductor es contundente,

cuando afirma que Betanzos «presumía de gran lenguaraz» (De la Vega, I960, IV: I40). No lo era; sólo presumía de ello.

RECIBIDO EN ENERO 2008 ACEPTADO EN FEBRERO 2008

\section{BIBLIOGRAFÍA}

Alvar, M. (1970). Americanismos en la «bistoria» de Bernal Díaz del Castillo. Centro Superior de Investigaciones Científicas, Revista de filología española, Anexo LXXXIX, Madrid.

Anónimo ([1586] 1951). Vocabulario y phrasis de la lengua general de los indios del Perú, llamada quichua y en la española. Lima: Ed. Fac., Instituto de Historia, UNMSM.

Bertonio, L. ([1612] I879). Vocabulario de la lengua Aymara. Teubner, Leipzig: Ed. Fac.

Betanzos, J. de ([155I] I987). Suma y narración de los Incas. María del Carmen Martín R. Madrid: Ed., Atlas.

Cieza de León, P. ([1550] 1989) Crónica del Perú. Tercera Parte. Edición, Prólogo y Notas por Francesca Cantù, $2^{a}$ Edición. Lima: Pontificia Universidad Católica.

Covarrubias, S. ([I6II] I995). Tesoro de la lengua castellana y española. Madrid: Ed. Castalia.

Corominas, J. (1983). Breve diccionario etimológico de la lengua castellana. $3^{\mathrm{a}}$. Ed. Madrid: Gredos.

Diccionario de la lengua española. (1992). Real Academia de la Lengua, $2 \mathrm{I}^{\mathrm{a}}$. Ed., Madrid.

Enguita Utrilla, J. M. (1996). «Indoamericanismos léxicos en algunos textos relativos a la conquista y colonización del Nuevo Mundo». Studia Neophilologica, Scandinavian University Press, Vol. LXVIII, No. 2, pp. 233-243.

- (1979). «Indoamericanismos léxicos en el Sumario de la Natural Historia de las Indias». Anuario de Letras, Facultad de Filosofía y Letras, Centro de Lingüística Hispánica, UNAM, México, pp. 285-304.

Fossa L. (2006). Narrativas problemáticas: Los Inkas bajo la pluma española. Lima: Instituto de Estudios Peruanos, Fondo Editorial de la Pontificia Universidad Católica del Perú y Author's Fund de la Universidad de Arizona. 
- (2003) http://www.coh.arizona.edu/spanish/FossaLydia/Betanzos/betanzos.htm

- (I998) «La apropiación de lo 'nuevo': la traducción». Actas del II Congreso Nacional de Investigaciones Lingüístico-Filológicas. Universidad Ricardo Palma, Facultad de Lenguas Modernas, Lima, Perú, pp. I6I-I7o.

Itier, C. (I995). «La littérature quechua d'évangélisation $\left(\mathrm{XVI}^{\mathrm{e}}\right.$ et $\mathrm{XVII}^{\mathrm{e}}$ siècles) comme source ethnolinguistique». Amérindia. Revue d'ethnolinguistique amérindienne. $\mathrm{Vol}$ I9/20, SELAF, Paris, pp. 321-33o.

- (I993) «Estudio y comentario lingüístico». Relacion de antiguedades deste reyno del Piru de Joan de Santa Cruz Pachacuti Yamqui Salcamaygua. Cuzco: IFEA-CBC, pp. I29-178.

Niranjana, T. (1992). Siting Translation. History, Post-Structuralism and the Colonial Context, U of California Press.

Pratt, M. L. (2002). "The Traffic in Meaning: Translation, Contagion, Infiltration». Profession 2002, Modern Language Association of America (MLA), Nueva York, pp. 25-36.
Pym, A. (2000). «On Method in Hispanic Translation History», Ponencia presentada en las $\mathrm{V}$ Jornadas Internacionales de Historia de la traducción, Universidad de León, 28-3I mayo.

Rafael, V. L. (1993). Contracting Colonialism. Translation and Christian Conversion in Tagalog Society Under Early Spanish Rule. Durham and London: Duke University Press.

Santo Tomás, D. de ([1560] 1951). Lexicon o vocabulario de la lengua general del Perú. Valladolid: Fernández de Córdova. Lima: Ed. Fac, UNMSM.

Schleiermacher, F. (I992). «From On the Different Methods of Translating». Theories of Translation. An Anthology of Essays from Dryden to Derrida de R. Schulte y J. Biguenet. Londres y Chicago: University of Chicago Press, pp. 36-54.

Venuti, L. (1993). «Translation as Cultural Politics: Regimes of Domestication in English». Textual Practice, Vol. 7, No. 2, Routledge, Summer, pp. 208-223. 